\title{
First Principles Study of Phonon Dispersion Curved for Yttrium Nitride
}

\author{
N.B. Yurdasan ${ }^{a}$, S.E. Gulebaglan ${ }^{a}$ And G.B. Akyuz ${ }^{b}$ \\ ${ }^{a}$ Dokuz Eylül University, Faculty of Sciences, Physics Department, 35160 Izmir, Turkey \\ ${ }^{b}$ Adnan Menderes University, Faculty of Sciences and Arts, Physics Department, 09100 Aydin, Turkey \\ We present first-principles calculations of the structural and lattice-dynamical properties for cubic yttrium \\ nitride. The ground state properties, such as the lattice constant and the bulk modulus, are calculated using \\ a plane wave pseudopotential method within density functional theory. A linear-response approach to density \\ functional theory is used to derive the phonon frequencies. In this work, we present plane-wave pseudopotential \\ calculations within density functional theory and calculate the phonon frequencies for high symmetry points.
}

DOI: 10.12693/APhysPolA.123.317

PACS: 71.15.Mb, 63.20.K-

\section{Introduction}

The electronic and optical properties of semiconductor compounds have been studied extensively in several works and well understood [1-4]. These structures are effectively used in heterostructures and quantum wells with various combinations. This is mainly due to their interesting optical properties used in high technology device production. Yttrium nitrate (YN) is explored as new compound for the technology applications. Only few theoretical papers and little experimental works have been devoted to study of structural and electronic properties of YN. The structural properties of YN have been determined by using the full potential linearized augmented plane wave (FP-LAPW) method in previous studies [5-8]. Transition metal nitrides which crystallize in the rock salt structure have wide range of technological applications, because of their high melting point, mechanical strength and high hardness $[9,10]$. In the literature, it was shown that $\mathrm{YN}$ is a semimetal with indirect band gap of $0.85 \mathrm{eV}$ and also, the stability of $\mathrm{YN}$ in the rock salt structure is determined with respect to zinc-blende and wurtzite structures [6].

In this study, there are reported the first principles calculations of the electronic, physical and dynamic properties of YN in the rock-salt structure which is an early transition metal mononitride and $4 d$ metal. In particular, lattice constant, bulk modulus, band structures, density of states, acoustic and optic phonon frequencies and phonon dispersion curve are reported. In Sect. 2 numerical method including the plane-wave pseudopotential (PP) code is given. The results are presented in Sect. 3 for the rock salt structure of YN. Finally, a brief conclusion is given in Sect. 4.

\section{Computational method}

The first principles pseudopotential method gives good agreement with experimental results and this method has been applied in variety of systems [11]. In this section, there are given only the computational details about the present calculations. The density functional theory with a plane-wave basis set as implemented in PWSCF [12] package program is employed for the calculations. The exchange and correlation effects have been treated using the generalized gradient approximation (GGA) [13, 14]. The electron-ion interactions are defined by ultrasoft pseudopotentials and these pseudopotentials are tested with the cut-off energy. Wave functions were expanded in a plane wave basis to a 40 Ry cutoff. A $10 \times 10 \times 10$ $k$-point grid has been used to sample the Brillouin zone. The chosen plane-wave cutoff and the number of $k$ point grid were carefully checked to ensure the total energy converged. Spin-orbit interactions were not included in calculation procedure. The bulk modulus and its pressure derivatives were calculated with Vinet et al. equation of states [15] using energy-volume variation. The root mean square error of energy obtained in fitting process was less than $1.0 \times 10^{-8} \mathrm{Ry}$ indicating high accuracy.

\section{Results}

The total energy of the YN was calculated as a function of the volume in the rock-salt phase using pseudopotential code. In this way, there are obtained the equilibrium lattice constant, bulk modulus, and other structural parameters. These parameters, which are obtained, are given in Table I which also includes some theoretical results and the available experimental data. The calculated lattice parameters and bulk modulus are in good agreement with the experimental data and the previous calculations. The calculated values for the ground state of rock-salt are $a_{0}=4.90 \AA$ and $B_{0}=160 \mathrm{GPa}$. The experimental lattice constant obtained $4.88 \AA$ is in agreement with the theory [16].

The electronic properties are crucial to the understanding of stability of the different structures. The band structure characteristic is important in the knowledge of changes in nearest-neighbor distances and symmetry under the pressure of YN. The self-consistent scalar rela- 
TABLE I

Calculated structural parameters for YN.

\begin{tabular}{c|c|c}
\hline \hline$a_{0}[\AA]$ & $B_{0}[\mathrm{GPa}]$ & $B^{\prime}$ \\
\hline $4.90^{*}$ & $160^{*}$ & $4.62^{*}$ \\
$4.92[5]$ & $157[5]$ & \\
$4.85[17]$ & $163[17]$ & \\
$4.88[6]$ & & \\
$4.91[7]$ & $154[7]$ & $3.06[7]$ \\
\hline
\end{tabular}

* Present work.

tivistic band structure of the $\mathrm{YN}$ is calculated in rock-salt phase along the various symmetry lines within the GGA scheme. As shown in Fig. 1, we found that YN is a semiconductor with indirect gap $\approx 0.22 \mathrm{eV}$. This result is in agreement with the results of Stampfl [6] who performed local density approximation calculations found that $\mathrm{YN}$ was a semiconductor with a indirect gap $\approx 1 \mathrm{eV}$.

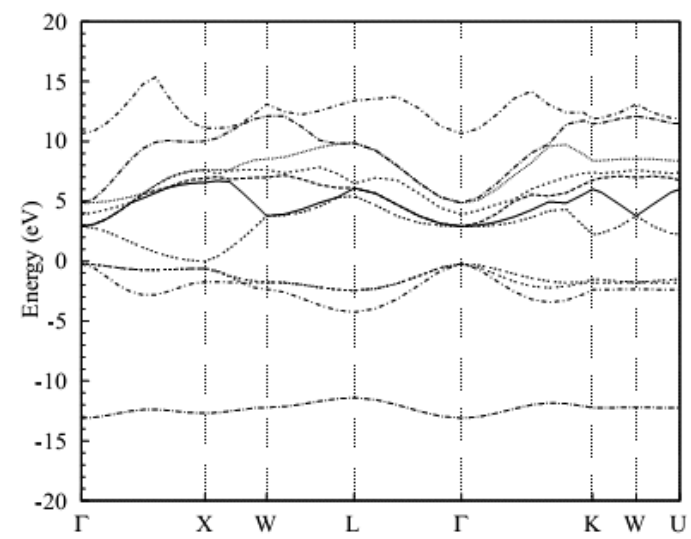

Fig. 1. Calculated electronic band structures for rock-salt phase of YN.

DOS and partial density of states (PDOS) of YN for GGA are also calculated and are given in Figs. 2, 3. The partial density of states clearly shows significant $p-d(\mathrm{~N} 2 p$ and $\mathrm{Y} 3 d$ ) hybridization near the Fermi surface $(12.22 \mathrm{eV})$ in the highest occupied molecular orbital (HOMO) and lowest unoccupied molecular orbital (LUMO) (Fig. 3). It can be said that there is a $p-d$ hybridization at valence and conduction bands and $p-d$ hybridization in HOMO is weaker than that in the LUMO. The lowest valence bands around $-10 \mathrm{eV}$ are entirely derived from the $p$ electrons of $\mathrm{Y}$, while the highest valence bands are characterized exclusively by the contributions of the $d$ orbitals of Y and $p$ orbitals of anion atoms. GGA phonon dispersion curves and density of states of YN along the high-symmetry directions were calculated by using the PWSCF.

The calculated phonon dispersion curves of $\mathrm{YN}$, along the principal symmetry direction of the Brillouin zone (BZ) are displayed in Fig. 4. The phonon dispersion of YN compounds are similar to $\mathrm{LaP}, \mathrm{LiF}, \mathrm{LiCl}$, and $\mathrm{NaBr}$ and the longitudinal acoustic (LA) and transversal acous-

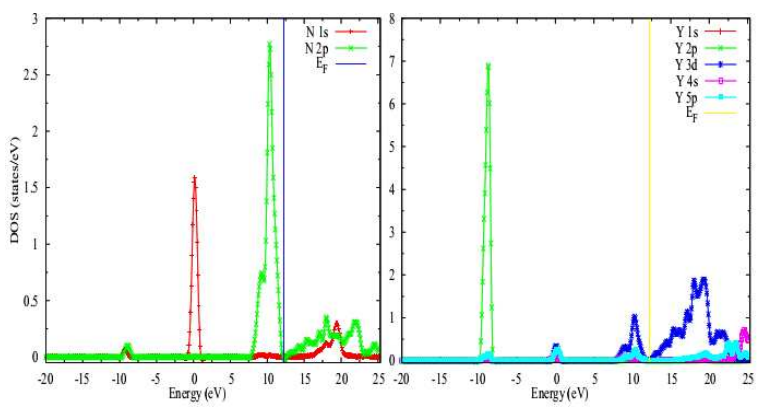

Fig. 2. Calculated partial density of states $\mathrm{N}$ and $\mathrm{Y}$ for rock-salt phase of $\mathrm{YN}$.

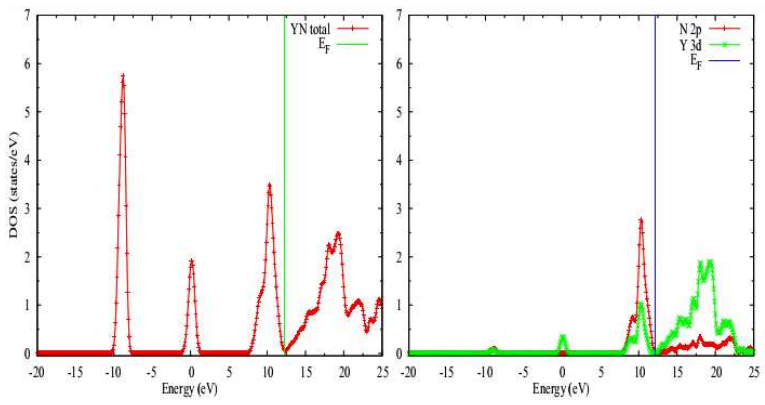

Fig. 3. Calculated density of states and partial density of states for rock-salt phase of $\mathrm{YN}$.

tic (TA) branches are clearly separated in the YN compounds. This feature is never seen in typical rock-salt materials $[16,18]$ however similar characteristic curves are observed in III-V compounds [19].

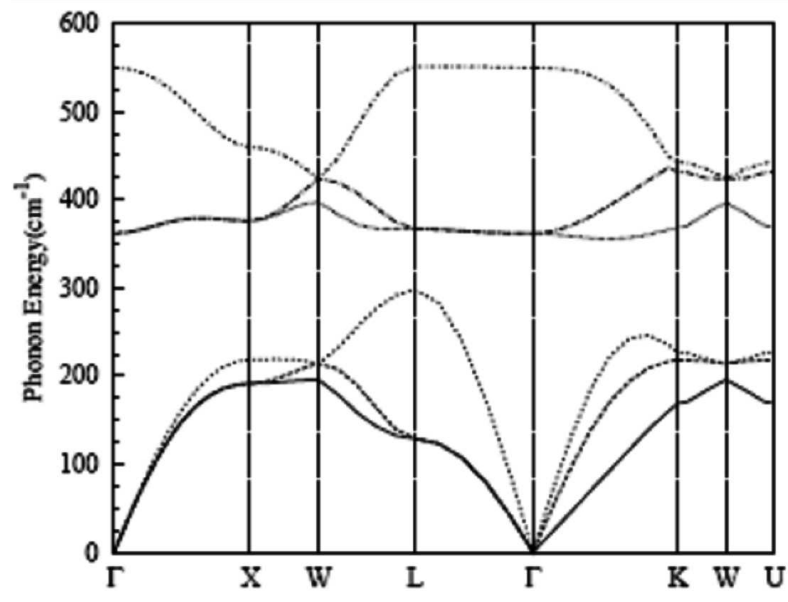

Fig. 4. Calculated phonon dispersion of YN rock salt.

The total and the projected density of phonon states are given in Fig. 5. The calculated phonon frequencies resulting from the high symmetry points of the BZ are listed in Table II. The optical branches do not show dispersive behavior and take place around $552 \mathrm{~cm}^{-1}$ frequency level for YN. 


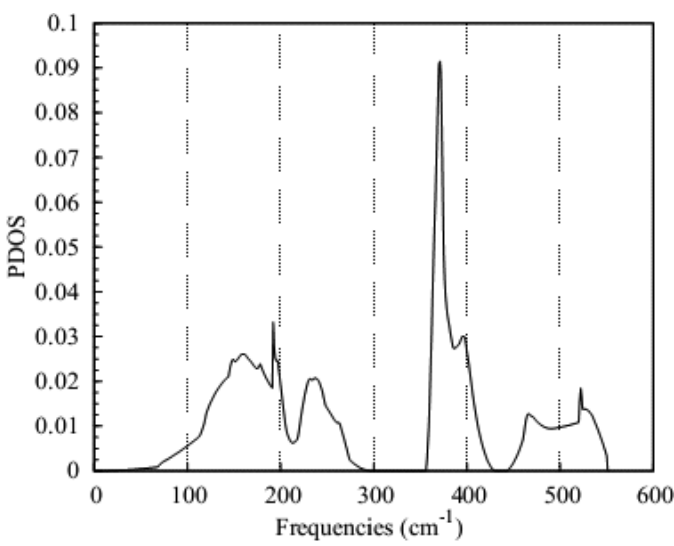

Fig. 5. Calculated phonon DOS of YN rock salt.

TABLE II

Phonon frequencies obtained at high symmetry points for YN.

\begin{tabular}{c|c|c|c}
\hline \hline & $\Gamma$ & $X$ & $L$ \\
\hline LA $\left[\mathrm{cm}^{-1}\right]$ & 0.0 & 219 & 298 \\
LO $\left[\mathrm{cm}^{-1}\right]$ & 549 & 461 & 369 \\
TA $\left[\mathrm{cm}^{-1}\right]$ & 0.0 & 191 & 128 \\
TO $\left[\mathrm{cm}^{-1}\right]$ & 362 & 378 & 552
\end{tabular}

The phonon DOS of YN show a sharp peak while the transverse optical (TO) phonon modes shows flatness along the $L \rightarrow \Gamma$ directions. The longitudinal optical (LO) phonon modes of YN also show flatness along the $L \rightarrow \Gamma$ directions, which gives a pronounced peak in the phonon DOS. The LA and TA branches of the semiconductor compound show flatness which includes a pronounced peak in the corresponding phonon DOS. The top of the bands for the LO phonon mode is not located at the zone center and the LO-TO splitting at the zone-centre is $187 \mathrm{~cm}^{-1}$ for YN. This splitting is similar to the other III-V semiconductors [16, 18].

\section{Conclusion}

In this report, $\mathrm{YN}$ in the rock salt structure is studied by using DFT to calculate ground state and dynamical properties. We have used GGA for exchange and correlation interaction. We obtained the lattice constants, phonon frequencies, bulk modulus and its derivatives of YN. Our results are in agreement with experimental data and theoretical values. We demonstrate that it is possible to predict the semiconductor material properties, such as lattice constant, band energy and phonon dispersion curved. Our results for the rock-salt structure in the presence of YN serve for a reference for future experimental work.

\section{References}

[1] M. Kumar, T. Nautiyal, S. Auluck, J. Phys., Condens. Matter 21, 446001 (2009)

[2] S. Berrah, A. Boukortt, H. Abid, Phys. Ser. 75, 414 (2007)

[3] M. Ferhat, A. Zaoui, Phys. Rev. B 73, 115107 (2006)

[4] Y. Xu, W.Y. Ching, Phys. Rev. B 48, 4335 (1993)

[5] L. Mancera, J.A. Rodriguez, N. Takeuchi, J. Phys., Condens. Matter 15, 2625 (2003)

[6] C. Stampfl, W. Mannstadt, R. Asahi, A.J. Freeman, Phys. Rev. B 63, 155106 (2001)

[7] W. De La Cruz, J.A. Diaz, L. Mancera, N. Takeuchi, G. Sato, J. Phys. Chem. Solids 64, 2273 (2003).

[8] B. Armani, F. El Haj Hassan, Comput. Mater. Sci. 39, 563 (2007)

[9] K. Inumaru, T. Ohara, S. Yamanaka, Appl. Surf. Sci. 158, 375 (2000)

[10] S. Yang, D.B. Lewis, I. Wadsworth, J. Cawley, J.S. Brooksadn, W.D. Munz, Surf. Coat. Technol. 131, 228 (2002)

[11] W.E. Pickett, Comput. Phys. Rep. 9, 115 (1989)

[12] S. Baroni, A. Dal Corso, S. de Gironcoli, P. Giannozzi, http://www.pwscf.org.

[13] J.P. Perdew, Y. Wang, Phys. Rev. B 45, 13244 (1992)

[14] J.P. Perdew, S. Burke, M. Ernzerhof, Phys. Rev. Lett. 77, 3865 (1992).

[15] P. Vinet, J. Ferrante, J. Smith, J. Rose, J. Phys. C 19, 467 (1986)

[16] A.M. Karo, J. Hardy, Phys. Rev. 129, 2024 (1963)

[17] N. Takeuchi, J. Phys. Rev. B 66, 153405 (2002).

[18] E. Deligöz, K. Çolakoğlu, Y.Ö. Ciftci, H. Özışı, J. Phys., Condens. Matter 19, 436204 (2007)

[19] A. Belabbes, A. Zaoui, M. Ferhat, J. Phys., Condens. Matter 20, 415221 (2008) 